Pacific

Journal of

Mathematics

\title{
ON PURIFIABLE SUBGROUPS AND THE INTERSECTION PROBLEM
}

\author{
TAKASHI OKUYAMA
}




\title{
ON PURIFIABLE SUBGROUPS AND THE INTERSECTION PROBLEM
}

\author{
TAKashi OKUYAMA
}

\begin{abstract}
L. Fuchs poses the problem of characterizing the subgroups of arbitrary abelian groups which are intersections of finitely many pure subgroups. We show that this problem for purifiable subgroups of primary abelian groups can be reduced to the case where the subgroups are vertical. We use this result to give a solution of this problem for subgroups of primary abelian groups in two special cases. Moreover, we obtain the following result: in a primary abelian group $G$, all pure hulls of a purifiable subgroup are $T$-high in $G$ for some subsocle $T$ of $G$.
\end{abstract}

Introduction. Problem 13 of L. Fuchs in his Infinite Abelian Groups, Academic Press, 1970, asks for a characterization of subgroups of an abelian group which are intersections of finitely many pure subgroups. This article is a contribution toward a solution of this difficult problem in the special case of primary groups with the additional assumption that the subgroups are already purifiable. We recall that a subgroup $A$ of a primary abelian group $G$ is said to be purifiable if it is contained in a minimal pure subgroup of $G$. Such a minimal pure subgroup is called a pure hull of $A$. We establish the rather unexpected fact that given a purifiable subgroup $A$ of a primary abelian group $G$ there exists a subsocle $T$ of $G$ such that all pure hulls of $A$ are $T$ high subgroups of $G$. This fact allows us to define two families of new relative invariants of purifiable subgroups. Next we show that the intersection problem for purifiable subgroups can be reduced to the case where the subgroups are also vertical subgroups. Vertical subgroups are studied extensively by [1] and can be defined thus: a subgroup $A$ of a primary abelian group $G$ is vertical in $G$ if

$$
\left(A+p^{n} G\right)[p]=A[p]+p^{n} G[p] \text { for all } n \geq 0 .
$$

We use these results to give a complete characterization of intersections of finitely many pure subgroups for subgroups $A$ in two special cases, namely when $A \cap p^{m} G[p]$ is a pure subgroup of $p^{m} G$ for some $m \geq 0$ and when $A \cap p^{m} G[p]$ is dense in $p^{m} G[p]$ for some $m \geq 0$.

All groups considered here are $p$-primary abelian groups for a fixed 
prime number $p$. The terminologies and notations not expressly introduced here follows the usage of [8]. Throughout this article, let $A$ be a subgroup of a group $G$.

\section{Purifiable subgroups.}

1.1. Definition. $A$ is said to be a purifiable subgroup of $G$ if, among the pure subgroups of $G$ containing $A$, there exists a minimal one. Such a minimal pure subgroup is called a pure hull of $A$ in $G$.

1.2. Definition. For every non-negative integer $n$, the $n$th overhang of $A$ in $G$ is the vector space

$$
V_{n}(G, A)=\left(\left(A+p^{n+1} G\right) \cap p^{n} G[p]\right) /\left(\left(A \cap p^{n} G[p]\right)+p^{n+1} G[p]\right) .
$$

It is convenient to use the following notations for the numerator and the denominator of $V_{n}(G, A)$ :

$$
A_{G}^{n}=\left(A+p^{n+1} G\right) \cap p^{n} G[p]=\left(\left(A \cap p^{n} G\right)+p^{n+1} G\right)[p]
$$

and

$$
A_{n}^{G}=\left(A \cap p^{n} G[p]\right)+p^{n+1} G[p]=A[p]_{G}^{n} .
$$

B. Charles was first to consider this notion in [7]. From [2], [4], and [11], the following statements hold:

1.3. Proposition. Let $A$ be a purifiable subgroup of $G$ and $H$ be a pure hull of $A$. Then the following properties hold:

(1) There exists a non-negative integer $m$ such that $V_{n}(G, A)=0$ for all $n \geq m$.

(2) $V_{n}(G, A) \simeq V_{n}(H, A)$ for every $n \geq 0$.

(3) $H=M \oplus N$, where $M$ and $N$ are subgroups of $H, M[p]=$ $A[p]$, and $N$ is bounded.

(4) $A$ is almost-dense in $H$.

A subgroup $A$ of $H$ is almost-dense in $H$ if $A+p^{n+1} H \supset p^{n} H[p]$ for all $n \geq 0$. The condition (1) is a necessary one for a subgroup $A$ to be purifiable in $G$. But, in general, this condition is not sufficient. For example, if $A$ is a subsocle of $G$, then $V_{n}(G, A)=0$ for all $n \geq 0$.

Finally, we want to characterize a subgroup of $G$ to be purifiable in $G$. Now we introduce the following invariant in [13].

1.4. Definition. For every non-negative integer $n$, the $n$th defect of $A$ in $G$ is the vector space

$$
D_{n}(G, A)=\left(p^{n}(G / A)[p]\right) /\left(\left(p^{n} G[p]+A\right) / A\right) .
$$


Similarly it is convenient to use the following notations for the numerator and the denominator of $D_{n}(G, A)$ :

$$
A^{n}(G)=p^{n}(G / A)[p]=\left(\left(p^{n} G+A\right) / A\right)[p]
$$

and

$$
A_{n}(G)=\left(p^{n} G[p]+A\right) / A .
$$

We use the above invariant to lead the following characterization of purity.

1.5. Proposition ([9, Proposition 3.2]). $A$ is pure in $G$ if and only if $D_{n}(G, A)=0$ for all $n \geq 0$.

Next we mention the relation between $V_{n}(G, A)$ and $D_{n}(G, A)$. The following properties are immediately proved by definitions and [13, Lemma 14].

1.6. Proposition. The following statements hold:

(1) $A_{n}(G) \cap A^{n+1}(G)=\left(\left(\left(A+p^{n+1} G\right) \cap p^{n} G[p]\right)+A\right) / A$ for every $n \geq 0$.

(2) For some integer $m \geq 0, V_{m}(G, A)=0$ if and only if $A_{m}(G) \cap$ $A^{m+1}(G)=A_{m+1}(G)$. 0 .

(3) For some integer $m \geq 0, D_{m+1}(G, A)=0$ implies $V_{m}(G, A)=$

(4) $A$ is neat in $G$ if and only if $D_{0}(G, A)=0$.

We use the $n$th defect of $A$ to provide a sufficient condition on a subgroup $A$ to be purifiable in $G$. But, in general, this condition is not necessary, although it implies the necessary conditions mentioned in Proposition 1.3 (1).

1.7. Proposition ([13, Theorem 3.1 and Theorem 3.2]). The following statements hold for some non-negative integer $m$.

(1) If $A_{n}(G)=A_{n+1}(G)$ for all $n \geq m$, then $A$ is purifiable in $G$.

(2) If $D_{n}(G, A)=0$ for all $n \geq m$, then $A$ is purifiable in $G$.

We recall a result from [13] that is used in this article.

1.8. LeMma ([13, Lemma 1.7]). Let $L$ be a pure subgroup of $G$ containing $A$. Then $A^{n}(G)=A^{n}(L)+A_{n}(G)$ and $A^{n}(L) \cap A_{n}(G)=$ $A_{n}(L)$ for every integer $n \geq 0$.

We also need the following concepts: 
1.9. Definition. $A$ is said to be an $m$-vertical subgroup of $G$ if there exists the least non-negative integer $m$ such that $V_{n}(G, A)=0$ for all $n \geq m$. If $m=0$, then $A$ is simply said to be vertical in $G$. Moreover, we say that a subgroup is eventually vertical in $G$ if it is $m$-vertical for some $m>0$.

Every subsocle of $G$ is immediately vertical in $G$. For more on these notions, see [1] and [4].

The following lemma is used in Proposition 1.11 .

1.10. Lemma. Let $A$ be purifiable in $G$. For a pure hull $H$ of $A$ in $G$ and a positive integer $m$, the following conditions are equivalent:

(1) $p^{m-1} H[p] \not \subset A$ and $p^{m} H[p] \subset A$.

(2) $A$ is $m$-vertical in $G$.

Proof. By Proposition $1.3(4), A$ is almost-dense in $H$. Hence, by Proposition $1.6(1)$ and (2), $A_{n}(H)=A_{n+1}(H)$ if and only if $V_{n}(G, A)=0$. If (1) is satisfied, then $A_{m-1}(H) \neq 0$ and $A_{n}(H)=0$ for all $n \geq m$. Therefore $V_{m-1}(H, A) \neq 0$ and $V_{n}(H, A)=0$ for all $m \geq n$. By Proposition $1.3(2)$, we have $V_{m-1}(G, A) \neq 0$ and $V_{n}(G, A)=0$ for all $n \geq m$. Hence (2) is satisfied.

Conversely, suppose that (2) is satisfied. By Proposition 1.3 (2), we have $V_{m-1}(H, A) \neq 0$ and $V_{n}(H, A)=0$ for all $n \geq m$. Hence $A_{m-1}(H) \neq A_{m}(H)$ and $A_{n}(H)=A_{n+1}(H)$ for all $n \geq m$. On the other hand, by Proposition 1.3 (3), there exists a non-negative integer $s$ such that $p^{s} H[p] \subset A$. Since $A_{n}(H)=0$ for all $n \geq s$, we have $s=m$.

Proposition 1.3, Lemma 1.8, and Lemma 1.10 combined lead to the following result:

1.11. Proposition. Let $A$ be purifiable and $m$-vertical in $G$ for some integer $m \geq 0$. Let $H$ be a pure hull of $A$ in $G$. Then there exist subgroups $M$ and $N$ of $H$ satisfying the following conditions:

(1) $H=M \oplus N$.

(2) $M[p]=A[p], p^{m-1} N \neq 0$, and $p^{m} N=0$. Moreover, $A^{n}(G)=A^{n}(H) \oplus A_{n}(G)$ for every integer $n \geq m$.

Proposition 1.11 leads to the following unexpected but useful result.

1.12. Theorem. If $A$ is purifiable in $G$, then there exists a subsocle $T$ of $G$ such that every pure hull of $A$ is $T$-high in $G$. 
Proof. By Proposition 1.3 (1), $A$ is $m$-vertical in $G$ for some nonnegative integer $m$. Let $H$ be a pure hull of $A$ in $G$, then note that $p^{m} H[p]=\left(A \cap p^{m} G\right)[p]$. We can write $p^{m} G[p]=\left(A \cap p^{m} G\right)[p] \oplus T_{m}$ for some subsocle $T_{m}$ of $G$. By [4, Theorem 1.7] and [2, Theorem 1.7], we have $A_{G}^{n}=A_{H}^{n}+A_{n}^{G}$ and $A+p^{n+1} H \supset p^{n} H[p]$ for all $n \geq 0$. Hence we have

$$
\begin{aligned}
p^{m-1} G[p] & =A_{G}^{m-1} \oplus S_{m-1}=\left(A_{H}^{m-1}+A_{m-1}^{G}\right) \oplus S_{m-1} \\
& =\left(p^{m-1} H[p]+p^{m} G[p]\right) \oplus S_{m-1} \\
& =p^{m-1} H[p] \oplus T_{m} \oplus S_{m-1}
\end{aligned}
$$

where $S_{m-1}$ is a subsocle of $G$. By finitely many steps, we have

$$
G[p]=H[p] \oplus T_{m} \oplus S_{m-1} \oplus \cdots \oplus S_{0}
$$

where $S_{i}$ is a subsocle of $G, 0 \leq i \leq m-1$. Put $T=T_{m} \oplus S_{m-1} \oplus$ $\cdots \oplus S_{0}$, then every pure hull of $A$ in $G$ is $T$-high in $G$.

1.13. Corollary. Let $H$ and $K$ be pure hulls of $A$ in $G$. Then we have

$$
\left(p^{n} G[p]\right) /\left(p^{n} H[p]\right) \simeq\left(p^{n} G[p]\right) /\left(p^{n} K[p]\right)
$$

for all $n \geq 0$.

Other aspects of purifiability of subgroups were taken up in [1], [2], [3], [4], [10], [11], and [13].

2. The $n$th covering and complemental dimensions. Throughout this section, let $H$ and $K$ be pure hulls of $A$ in $G$. In this section, we point out some properties of $H / A$ and $G / H$. We use Theorem 1.12 and these properties to introduce new invariants of subgroups namely, the $n$th covering and $n$th complemental dimensions. These invariants play an important role in the intersection problem.

2.1. Proposition ([13, Theorem 4.3]). If $A$ is purifiable in $G$, then

$$
p^{n}(H / A)[p] \simeq p^{n}(K / A)[p]
$$

for all $n \geq 0$.

2.2. Definition. Let $A$ be purifiable in $G$. For every integer $n \geq 0$, we denote the dimension of $p^{n}(H / A)[p]$ as a vector space over the field of $p$-elements by

$$
\operatorname{Cov}_{n}(G, A)=\operatorname{dim} p^{n}(H / A)[p] .
$$


$\operatorname{Cov}_{n}(G, A)$ is called the $n$th covering dimension of $A$ in $G$. Proposition 2.1 shows that the $n$th covering dimension does not depend on the choice of a pure hull of $A$ and thus it is a relative invariant of $A$ in $G$.

In [5], K. Benabdallah and S. Robert defined $\operatorname{Def}(A)=\operatorname{dim}(N / A)[p]$ where $N$ is a neat hull of $A$ in $G$. If $N$ is pure in $G$, then $N$ is vertical in $G$ and this definition coincides with our $\operatorname{Cov}_{0}(G, A)$.

Recall that the $n$th Ulm Kaplansky invariant of a group $G$ is

$$
f_{n}(G)=\left(p^{n} G[p]\right) /\left(p^{n+1} G[p]\right) \text {. }
$$

2.3. Proposition [13, Proposition 4.1]). If $A$ is purifiable in $G$, then

$$
f_{n}(H / A) \simeq f_{n}(K / A)
$$

for all $n \geq 0$.

2.4. Definition. Let $A$ be purifiable in $G$. For every integer $n \geq 0$, we denote the dimension of $\left(p^{n} G[p]\right) /\left(p^{n} H[p]\right)$ by

$$
\operatorname{Com}_{n}(G, A)=\operatorname{dim}\left(p^{n} G[p]\right) /\left(p^{n} H[p]\right) .
$$

$\operatorname{Com}_{n}(G, A)$ is called the $n$th complemental dimension of $A$ in $G$. By Corollary 1.13, $\operatorname{Com}_{n}(G, A)$ does not depend on the choice of a pure hull of $A$ in $G$. Thus it is also a relative invariant of $A$ in $G$. Moreover, since $H$ is pure in $G$, we have $\left(p^{n} G[p]\right) /\left(p^{n} H[p]\right) \simeq$ $p^{n}(G / H)[p]$.

Recall that the $n$th relative Ulm Kaplansky invariant of $A$ in $G$ is

$$
U_{n}(G, A)=\left(p^{n} G[p]\right) /\left(\left(A+p^{n+1} G\right) \cap p^{n} G[p]\right)=\left(p^{n} G[p]\right) / A_{G}^{n} .
$$

In a discussion with $\mathrm{K}$. Benabdallah, we obtained the following result. Before we do this, we need the so-called Dedekind short exact sequence.

2.5. LemMA. Let $T, S, U$ be submodules of a module $G$ over some ring such that $T \subset S$. Then there exists the following short exact sequence:

$$
0 \rightarrow(S \cap U) /(T \cap U) \rightarrow S / T \rightarrow(S+U) /(T+U) \rightarrow 0 .
$$

In particular, $(S \cap U) /(T \cap U) \simeq S / T$ if and only if $(S \cap U)+T=S$, and $S / T \simeq(S+U) /(T+U)$ if and only if $S \cap U \subset T$.

2.6. Proposition. If $A$ is purifiable in $G$, then

$$
f_{n}(G / H) \simeq f_{n}(G / K)
$$

for all $n \geq 0$. 
Proof. By [4, Theorem 1.7] and [2, Theorem 1.7], it follows that $A_{G}^{n}=A_{H}^{n}+A_{n}^{G}$ and $A+p^{n+1} H \supset p^{n} H[p]$ for all $n \geq 0$. Hence we have $U_{n}(G, A)=\left(p^{n} G[p]\right) /\left(p^{n} H[p]+p^{n+1} G[p]\right)$. On the other hand, we have

$$
\begin{aligned}
f_{n}(G / H) & =\left(p^{n}(G / H)[p]\right) /\left(p^{n+1}(G / H)[p]\right) \\
& \simeq\left(p^{n} G[p]+H\right) /\left(p^{n+1} G[p]+H\right) \\
& =\left(p^{n} G[p]+p^{n} H[p]+H\right) /\left(p^{n+1} G[p]+p^{n} H[p]+H\right) .
\end{aligned}
$$

Put $S=p^{n} G[p]+p^{n} H[p], T=p^{n+1} G[p]+p^{n} H[p]$, and $U=H$. Since $S \cap U \subset T$, we have

$$
f_{n}(G / H) \simeq\left(p^{n} G[p]\right) /\left(p^{n} H[p]+p^{n+1} G[p]\right) \simeq U_{n}(G, A)
$$

by Lemma 2.5. Hence it follows that $f_{n}(G / H) \simeq U_{n}(G, A) \simeq f_{n}(G / K)$ for all $n \geq 0$.

We use the Dedekind short exact sequence to prove the following lemma that is used in Lemma 3.1.

2.7. Lemma. Let $L$ be a pure subgroup of $G$ containing $A$. Then

$$
A^{n}(G) / A^{n}(L) \simeq p^{n} G[p] / p^{n} L[p]
$$

for every integer $n \geq 0$.

Proof. By Lemma 1.8, note that

$$
\begin{aligned}
A^{n}(G) / A^{n}(L) & =\left(A^{n}(L)+A_{n}(G)\right) / A^{n}(L) \\
& \simeq A_{n}(G) /\left(A^{n}(L) \cap A_{n}(G)\right) \simeq A_{n}(G) / A_{n}(L) \\
& \simeq\left(p^{n} G[p]+A\right) /\left(p^{n} L[p]+A\right) .
\end{aligned}
$$

Put $S=p^{n} G[p], T=p^{n} L[p]$, and $U=A$. Since $S \cap A=p^{n} G[p] \cap$ $A \subset L \cap p^{n} G[p]=p^{n} L[p]=T$, we have $\left(p^{n} G[p]+A\right) /\left(p^{n} L[p]+A\right) \simeq$ $p^{n} G[p] / p^{n} L[p]$ by Lemma 2.5. Hence $A^{n}(G) / A^{n}(L) \simeq p^{n} G[p] / p^{n} L[p]$ for every integer $n \geq 0$.

3. The Intersection Problem. L. Fuchs poses the problem of characterizing the subgroups of arbitrary abelian groups, which are intersections of finitely many pure subgroups ([8] Problem 13, p. 134). We call this problem the Intersection Problem.

C. Megibben characterized intersections of pure subgroups of primary groups in [12]. D. Boyer and K. M. Rangaswamy characterized 
such subgroups in arbitrary abelian groups in [6]. R. Göbel and R. Vergohen characterized intersections of pure subgroups of valuated abelian groups in [9]. K. Benabdallah and S. Robert gave a characterization of intersections of finitely many neat subgroups in primary abelian groups in [5]. Moreover, under the condition that $A[p]$ is dense in $G[p]$, they solved the intersection problem in [5]. However, nothing is known about intersections of finitely many pure subgroups except the fact mentioned above.

It is well known that if $A[p]$ is dense in $G[p]$, then $A$ is purifiable in $G$. In this article, as the first step toward solving the intersection problem, we study the problem for subgroups which are already purifiable. Before we give necessary conditions for subgroups to be intersections of finitely many pure subgroups in given groups, we need the following lemmas.

3.1. Lemma. Let $M$ be a non-negative integer. Let $A$ be $m$ vertical and purifiable in $G$ and let $L$ be a pure subgroup of $G$ containing $A$. Then

$$
\operatorname{dim} A^{n}(G) / A^{n}(L) \leq \operatorname{Com}_{n}(G, A)
$$

for every integer $n \geq 0$.

Proof. Let $H$ be a pure hull of $A$ in $G$. Note that $p^{m} H[p]=$ $\left(A \cap p^{m} G\right)[p]$ and $p^{m} G[p]=\left(A \cap p^{m} G\right)[p] \oplus T_{m}$ for some subsocle $T_{m}$ of $G$. For every $n \geq m$, since $p^{n} L[p]=\left(A \cap p^{n} G\right)[p] \oplus\left(L \cap T_{n}\right)$, we have $p^{n} G[p]=p^{n} L[p] \oplus T_{n}^{\prime}$ for some subgroup $T_{n}^{\prime}$ of $T_{n}$. By Lemma 2.7, we have $A^{n}(G) / A^{n}(L) \simeq p^{n} G[p] / p^{n} L[p] \simeq T_{n}^{\prime} \subset T_{n} \simeq$ $\operatorname{Com}_{n}(G, A)$. Hence we may assume that $n<m$. By the proof of Theorem 1.12, it follows that

$$
\begin{aligned}
p^{m-1} G[p] & =A_{G}^{m-1} \oplus S_{m-1}=\left(A_{L}^{m-1}+p^{m} G[p]\right) \oplus S_{m-1} \\
& =\left(\left(A_{L}^{m-1}+\left(A \cap p^{m} G\right)[p]\right) \oplus T_{m}\right) \oplus S_{m-1}
\end{aligned}
$$

where $S_{m-1}$ is a subsocle of $G$. Hence we have $p^{m-1} G[p]=p^{m-1} L[p]$ $\oplus R_{m-1}$ for some subgroup $R_{m-1}$ of $T_{m} \oplus S_{m-1}$. Since $p^{m-1} G[p]=$ $p^{m-1} H[p] \oplus T_{m} \oplus S_{m-1}$, our assertion holds for $n=m-1$ by Lemma 2.7. By finitely many steps, we have $\operatorname{dim} A^{n}(G) / A^{n}(L) \leq \operatorname{Com}_{n}(G, A)$ for every $n<m$.

3.2. Lemma. Let $A$ be purifiable in $G$. If $A$ is an intersection of finitely many pure subgroups in $G$, then there exists a positive integer $t$ such that

$$
\operatorname{Cov}_{0}(G, A) \leq t \operatorname{Com}_{0}(G, A) \text {. }
$$


Proof. Let $A=\bigcap_{i=1}^{t} H_{i}$ where $H_{i}$ is a pure subgroup of $G$ containing $A$. Put $H_{0}=H$ and $W_{s}=\bigcap_{i=0}^{s} A^{0}\left(H_{i}\right)$. Then we have

$$
\operatorname{Cov}_{0}(G, A)=\sum_{i=0}^{t-1} \operatorname{dim}\left(W_{s} / W_{s+1}\right) \text {. }
$$

Since $W_{s} / W_{s+1}=W_{s} /\left(W_{s} \cap A^{0}\left(H_{s+1}\right)\right) \simeq\left(W_{s}+A^{0}\left(H_{s+1}\right)\right) / A^{0}\left(H_{s+1}\right) \subset$ $A^{0}(G) / A^{0}\left(H_{s+1}\right)$, by Lemma 3.1, we have

$$
\operatorname{dim} W_{s} / W_{s+1} \leq \operatorname{dim} A^{0}(G) / A^{0}(H)=\operatorname{Com}_{0}(G, A) .
$$

Hence it follows that $\operatorname{Cov}_{0}(G, A) \leq t \operatorname{Com}_{0}(G, A)$.

3.3. Lemma. Let $A$ be purifiable in $G$. Then, for every integer $n \geq 0, \operatorname{Cov}_{n}(G, A)=\operatorname{Cov}_{0}\left(p^{n} G, p^{n} G \cap A\right)$ and $\operatorname{Com}_{n}(G, A)=$ $\operatorname{Com}_{0}\left(p^{n} G, p^{n} G \cap A\right)$.

Proof. Let $H$ be a pure hull of $A$ in $G$. By [1, Theorem 5.3], $p^{n} H$ is a pure hull of $A \cap p^{n} G$ in $p^{n} G$ and $A \cap p^{n} G=A \cap p^{n} H$. Hence we have $\operatorname{Cov}_{0}\left(p^{n} G, p^{n} G \cap A\right)=\operatorname{dim}\left(p^{n} H /\left(p^{n} H \cap A\right)\right)[p]=\operatorname{dim} A^{n}(H)=$ $\operatorname{Cov}_{n}(G, A)$ and $\operatorname{Com}_{0}\left(p^{n} G, p^{n} G \cap A\right)=\operatorname{dim}\left(p^{n} G[p] / p^{n} H[p]\right)=$ $\operatorname{Com}_{n}(G, A)$.

3.4. TheOREM. Let $A$ be purifiable in $G$. If $A$ is an intersection of finitely many pure subgroups in $G$, then, for every integer $n \geq 0$, there exists a positive integer $t_{n}$ such that

$$
\operatorname{Cov}_{n}(G, A) \leq t_{n} \operatorname{Com}_{n}(G, A) .
$$

Proof. Let $A=\bigcap_{i=1}^{t} H_{i}$ where $H_{i}$ is a pure subgroup of $G$ containing $A$. By [1, Theorem 5.3], $A \cap p^{n} G$ is purifiable in $p^{n} G$. Moreover, we have $A \cap p^{n} G=\bigcap_{i=1}^{t}\left(H_{i} \cap p^{n} G\right)=\bigcap_{i=1}^{t} p^{n} H_{i}$ and $p^{n} H_{i}$ is a pure subgroup of $p^{n} G$ containing $A \cap p^{n} G$. Hence, by Lemma 3.2 and Lemma 3.3, there exists a positive integer $t_{n}$ such that $\operatorname{Cov}_{n}(G, A) \leq t_{n} \operatorname{Com}_{n}(G, A)$.

Theorem 3.4 gives necessary conditions on a purifiable subgroup to be an intersection of finitely many pure subgroups in a given group. It is natural to consider whether these conditions are sufficient. But, since the Intersection Problem is difficult, we do not know the answer at the present time. However, from Theorem 3.6, the author has a conjecture that these conditions are sufficient for a vertical subgroup.

Let $A$ be purifiable and $m$-vertical in $G$ for some positive integer $m$. If $A$ is an intersection of finitely many pure subgroups in $G$, then 
$A \cap p^{m} G$ is an intersection of finitely many pure subgroups in $p^{m} G$ and $A$ satisfies the condition in Theorem 3.4. If the converse of the fact mentioned above is true, then we may assume that $A$ is vertical in $G$ when we study the Intersection Problem for subgroups which are already purifiable.

First, we need the following lemma.

3.5. LemMA. Let $A$ be purifiable and $m$-vertical in $G$ for some positive integer $m$, and let $H$ be a pure hull of $A$ in $G$. Let $A^{0}(H)=$ $A^{m} \oplus A^{m}(H)$ where $A^{m}$ is a subsocle of $A^{0}(H)$. Moreover, put $A^{m}=$ $\bigoplus_{\lambda \in \Lambda}\left\langle h_{\lambda}+A\right\rangle$ and $E=\left\{h_{\lambda} \mid \lambda \in \Lambda\right\}$. Let $T$ be a subsocle of $G$ where every pure hull of $A$ in $G$ is a T-high subgroup of $G$ and $F$ be a set of basis of $T$. If $\operatorname{Cov}_{0}(G, A) \leq \operatorname{Com}_{0}(G, A)$, then there exists a pure hull $K$ of $A$ in $G$ such that $K / A \cap A^{m}=0$.

Proof. By hypothesis, there exists an injection $\Psi: E \rightarrow F$. Let $H_{m}=\bigoplus_{\lambda \in \Lambda}\left\langle h_{\lambda}+\Psi\left(h_{\lambda}\right)+A ; \lambda \in \Lambda\right\rangle$, then $H_{m} / A \cap A^{m}(G)=0$. To show this, let $h+x+A \in H_{m} / A \cap A^{m}(G)$ where $h \in H$ and $x \in T$. Then we may assume that $h_{G / A}(h+A)=n<m$. By Proposition 1.11 and Theorem 1.12, we have $A^{m}(G)=A^{m}(H) \oplus A_{m}(G)$ and $p^{m} G[p]=$ $p^{m} H[p] \oplus T_{m}$ where $T_{m}=T \cap p^{m} G[p]$. Hence it follows that

$$
x=a-h+p^{m} h_{1}+a_{m}+t_{m}
$$

where $a \in A, p^{m} h_{1} \in p^{m} H, p^{m+1} h_{1} \in A, a_{m} \in\left(A \cap p^{m} G\right)[p]=$ $p^{m} H[p]$, and $a_{m} \in T_{m}$. Since $G[p]=H[p] \oplus T$, we have $h-a=$ $p^{m} h_{1}+a_{m}$. Hence $h+A \in A^{m}(H)$. This is a contradiction.

Since $H_{m} \cap\left(p^{m} G+A\right)=\left(H_{m} \cap p^{m} G\right)+A \subset A$, it follows that $H_{m} \cap p^{m} G=A \cap p^{m} G$. Hence it follows that $\left(H_{m}+p^{m} H\right) \cap p^{m} G=$ $\left(H_{m} \cap p^{m} G\right)+p^{m} H=p^{m} H$. By [1, Proposition 2.9], we can extend $H_{m}+p^{m} H$ to a pure subgroup $K$ of $G$ such that $K \cap p^{m} G=$ $p^{m} H$. Moreover, by [2, Theorem 2.1] there exists $t>0$ such that $p^{t+m} H[p] \subset p^{m} G \cap A$. Thus, since $p^{t+m} K[p] \subset A, K$ is a pure hull of $A$ in $G$. Furthermore we have $K / A \cap A^{m}=0$.

From Lemma 3.5, we establish the following reduction theorem:

3.6. Theorem. Let $A$ be purifiable and $m$-vertical in $G$ for some positive integer $m$. $A$ is an intersection of finitely many pure subgroups in $G$ if and only if the following conditions hold:

(1) $p^{m} G \cap A$ is an intersection of finitely many pure subgroups in $p^{m} G$. 
(2) There exists a positive integer $t$ such that

$$
\operatorname{Cov}_{0}(G, A) \leq t \operatorname{Com}_{0}(G, A) .
$$

Proof. The necessity is immediate. Conversely, suppose that the conditions hold. Let $H$ be a pure hull of $A$ in $G$. Then we can write

$$
A \cap p^{m} G=\left(\bigcap_{i=1}^{t} L_{i}\right) \cap p^{m} H
$$

where $L_{i}$ is a pure subgroup of $p^{m} G$ containing $A \cap p^{m} G, 1 \leq i \leq t$. Since $\left(A+L_{i}\right) \cap p^{m} G=L_{i}$, we can extend $A+L_{i}$ to a pure subgroup $M_{i}$ of $G$ satisfying $M_{i} \cap p^{m} G=p^{m} M_{i}=L_{i}$ by [1, Proposition 2.9]. Then $A^{m}(H) \cap\left(\bigcap_{i=1}^{t} A^{0}\left(M_{i}\right)\right)=0$. Because, we have

$$
\begin{aligned}
A^{m}(H) \cap\left(\bigcap_{i=1}^{t} A^{0}\left(M_{i}\right)\right)=A^{m}(H) \cap\left(\bigcap_{i=1}^{t}\left(A^{0}\left(M_{i}\right) \cap A^{m}(G)\right)\right) \\
=A^{m}(H) \cap\left(\bigcap_{i=1}^{t} A^{0}\left(L_{i}\right)\right) \\
\subset\left(\left(p^{m} H+A\right) / A\right) \cap\left(\bigcap_{i=1}^{t}\left(L_{i} / A\right)\right) \\
=\left(\left(\left(p^{m} H\right) \cap\left(\bigcap_{i=1}^{t} L_{i}\right)\right)+A\right) / A=0 .
\end{aligned}
$$

Note that $A^{0}(H)=A^{m} \oplus A^{m}(H)$ where $A^{m}$ is defined in Lemma 3.5. If $\operatorname{Com}_{0}(G, A)$ is infinite, then $\operatorname{Cov}_{0}(G, A) \leq \operatorname{Com}_{0}(G, A)$ by hypothesis. By Lemma 3.5, there exists a pure hull $K$ of $A$ in $G$ such that $K / A \cap A^{m}=0$. Hence we have $K \cap\left(\bigcap_{i=1}^{t} M_{i}\right) \cap H=A$.

Next, suppose that $\operatorname{Com}_{0}(G, A)$ is finite. Then $\operatorname{Cov}_{0}(G, A)$ is finite by hypothesis. Hence $A^{m}$ is finite. By a similar argument to Lemma 3.5, there exists a pure hull $K_{\lambda}$ of $A$ in $G$ such that $K_{\lambda} / A \cap$ $\left\langle h_{\lambda}+A\right\rangle=0$. Hence $\left(\bigcap_{\lambda \in \Lambda} K_{\lambda}\right) \cap H \cap\left(\bigcap_{i=1}^{t} M_{i}\right)=A$, and so $A$ is an intersection of finitely many pure subgroups in $G$.

With our notation, C. Megibben's result in [12] can be formulated as follows:

3.7. Proposition. $A$ is an intersection of pure subgroups in $G$ if and only if the following condition holds: 


$$
A_{n}(G)=\left(p^{n} G[p]+A\right) / A=0 \quad \text { implies } A^{n}(G)=p^{n}(G / A)[p]=0
$$

for every non-negative integer $n$.

Suppose that $A$ satisfies Megibben's condition. If $A_{m}(G)=0$ for some integer $m$, then we have $A^{m}(G)=0$. The condition that $D_{n}(G, A)=0$ for all $n \geq m$ is weaker than $A^{m}(G)=0$. By Proposition 1.7 (2), if $D_{n}(G, A)=0$ for all $n \geq m$, then $A$ is purifiable in $G$ and $A$ satisfies Megibben's condition.

Now, under the condition that $D_{n}(G, A)=0$ for all $n \geq m$, we give necessary and sufficient conditions for a subgroup $A$ to be an intersection of finitely many pure subgroups in $G$. Before we do this, we need the following lemma.

3.8. LeMMA. Let $H$ be a pure hull of $A$ in $G$ and $m$ be a positive integer. If $D_{n}(G, A)=0$ for every $n \geq m$, then $A^{0}(G)=A^{0}(H) \oplus$ $A_{m}(G)$ and $\operatorname{Com}_{0}(G, A)=\operatorname{Com}_{m}(G, A)$.

Proof. Let $H$ be a pure hull of $A$ in $G$. Then $p^{m} H=A \cap p^{m} G$ by [1, Theorem 5.3]. Hence $A^{m}(H)=0$, and so $H / A$ is an $A^{m}(G)$-high subgroup of $G / A$. We have $A^{0}(G)=A^{0}(H) \oplus A^{m}(G)=A^{0}(H) \oplus$ $A_{m}(G)$ and $\operatorname{Com}_{0}(G, A)=\operatorname{Com}_{m}(G, A)$.

Theorem 3.4, Theorem 3.6, and Lemma 3.8 combined lead to the following corollary.

3.9. Corollary. Suppose $D_{n}(G, A)=0$ for every $n \geq m$ where $m$ is some positive integer. Then $A$ is an intersection of finitely many pure subgroups in $G$ if and only if there exists a positive integer $t$ such that

$$
\operatorname{Cov}_{0}(G, A) \leq t \operatorname{Com}_{m}(G, A) .
$$

Note that $D_{n}(G, A)=0$ for every $n \geq m$ where $m>0$ is equivalent to the fact that $A \cap p^{m} G$ is pure in $p^{m} G$.

On the other hand, the condition that $A[p]$ is dense in $G[p]$ implies $A_{n}(G)=A_{n+1}(G)$ for all $n \geq 0$. It is immediate that this condition is stronger than $A_{n}(G)=A_{n+1}(G)$ for all $n \geq m$ where $m$ is some positive integer. By Proposition $1.7(1)$, if $A_{n}(G)=A_{n+1}(G)$ for all $n \geq m$, then $A$ is purifiable in $G$ and $A$ satisfies Megibben's condition.

Next, under the condition that $A_{n}(G)=A_{n+1}(G)$ for all $n \geq m$, we give necessary and sufficient conditions for a subgroup $A$ to be an intersection of finitely many pure subgroups in $G$. 
Theorem 3.4, Theorem 3.6, and [5, Theorem 3.3] combined lead to the following corollary.

3.10. COROLlary. Suppose there exists a positive integer $m$ such that $A_{n}(G)=A_{n+1}(G)$ for all $n \geq m$. Then $A$ is an intersection of finitely many pure subgroups in $G$ if and only if there exist positive integers $t_{0}$ and $t_{m}$ such that $\operatorname{Cov}_{0}(G, A) \leq t_{0} \operatorname{Com}_{0}(G, A)$ and $\operatorname{Cov}_{m}(G, A) \leq t_{m} \operatorname{Com}_{m}(G, A)$.

Note that $A_{n}(G)=A_{n+1}(G)$ for every $n \geq m$ where $m>0$ is equivalent to the fact that $A \cap p^{m} G[p]$ is dense in $p^{m} G[p]$.

Recall that $A$ is said to be almost-dense in $G$ if $A_{n}(G) \subset A^{n+1}(G)$ for all $n \geq 0$.

By Corollary 3.10, we immediately state the following:

3.11. Corollary. Let $A$ be not pure, purifiable, and almost-dense in $G$. $A$ is an intersection of finitely many pure subgroups in $G$ if and only if there exist positive integers $m, t_{0}$, and $t_{m}$, such that $\operatorname{Cov}_{0}(G, A)$ $\leq t_{0} \operatorname{Com}_{0}(G, A)$ and $\operatorname{Cov}_{m}(G, A) \leq t_{m} \operatorname{Com}_{m}(g, A)$.

Acknowledgment. The author wishes to express his grateful thanks to Professor K. Benabdallah for his indicating some corrections and his help during the preparation of this manuscript.

\section{REFERENCES}

[1] K. Benabdallah, C. Charles, and A. Mader, Vertical subgroups of primary groups, Canad. J. Math., 43 (1) (1991), 3-18.

[2] K. Benabdallah and J. Irwin, On minimal pure subgroups, Publ. Math. Debrecen, 23 (1976), 111-114.

[3] K. Benabdallah and C. Piché, Sous-groupes purifiables des groupes abéliens primaires, Canad. Bull. Math., 32 No. 4 (1989), 11-17.

[4] K. Benabdallah and T. Okuyảma, On purifiable subgroups of primary abelian groups, Comm. Algebra, 19 (1) (1991), 85-96.

[5] K. Benabdallah and S. Robert, Intersections finies de sous-groupes nets, Canad. J. Math., 32 No. 4 (1980), 885-892.

[6] D. Boyer and K. M. Rangaswamy, Intersections of pure subgroups in abelian groups, Proc. Amer. Math. Soc., 81 No. 2 (1981), 178-180.

[7] B. Charles, Etudes sur les sous-groupes d'un groupe abélien, Bull. Soc. Math. France, 88 (1960), 217-227.

[8] L. Fuchs, Infinite Abelian Groups, I, II, Academic Press, 1970 and 1973.

[9] R. Göbel and R. Vergohen, Intersections of pure subgroups of valuated abelian groups, Arch. Math., 39 (1982), 525-534.

[10] T. Head, Remarks on a problem in primary abelian groups, Bull. Soc. Math. France, 91 (1963), 102-112. 
[11] P. Hill and C. Megibben, Minimal pure subgroups in primary groups, Bull. Soc. Math. France, 92 (1964), 251-257.

[12] C. Megibben, On subgroups of primary abelian groups, Publ. Math. Debrecen, 12 (1965), 293-294.

[13] T. Okuyama, On the existence of pure hulls in primary abelian groups, Comm. Algebra, 19 (11) (1991), 3089-3098.

Received January 24, 1991 and in revised form October 8, 1991.

Toba National College of Maritime Technology 1-1, IKegami-Cho, Toba-SHI

MIE-KEN, 517, JAPAN 


\title{
PACIFIC JOURNAL OF MATHEMATICS
}

\author{
Founded by
}

E. F. BECKENBACH (1906-1982) F. WoLF (1904-1989)

\section{EDITORS}

$\begin{array}{ll}\begin{array}{l}\text { V. S. VARADARAJAN } \\ \text { (Managing Editor) }\end{array} & \begin{array}{l}\text { NiCHOLAS ERCOLANI } \\ \text { University of Arizona }\end{array} \\ \text { University of California } & \text { Tucson, AZ 85721 } \\ \text { Los Angeles, CA 90024-1555 } & \text { ercolani@math.arizona.edu } \\ \text { vsv@math.ucla.edu } & \text { R. FinN } \\ \text { HERBERT CLEMENS } & \text { Stanford University } \\ \text { University of Utah } & \text { Stanford, CA 94305 } \\ \text { Salt Lake City, UT 84112 } & \text { finn@gauss.stanford.edu } \\ \text { clemens@math.utah.edu } & \text { VAUGHAN F. R. JoNEs } \\ \text { F. MichAEL CHRIsT } & \text { University of California } \\ \text { University of California } & \text { Berkeley, CA 94720 } \\ \text { Los Angeles, CA 90024-1555 } & \text { vfr@math.berkeley.edu } \\ \text { christ@math.ucla.edu } & \text { STEVEN KERCKHoFF } \\ \text { THomas ENRIGHT } & \text { Stanford University } \\ \text { University of California, San Diego } & \text { Stanford, CA 94305 } \\ \text { La Jolla, CA 92093 } & \text { spk@gauss.stanford.edu } \\ \text { tenright@ucsd.edu } & \end{array}$

\author{
C. C. MOORE \\ University of California \\ Berkeley, CA 94720
}

MaRTin ScharlemanN

University of California

Santa Barbara, CA 93106

mgscharl@henri.ucsb.edu

\author{
HAROLD STARK \\ University of California, San Diego \\ La Jolla, CA 92093
}

\begin{tabular}{ll}
\multicolumn{1}{c}{ SUPPORTING } & INSTITUTIONS \\
UNIVERSITY OF ARIZONA & UNIVERSITY OF OREGON \\
UNIVERSITY OF BRITISH COLUMBIA & UNIVERSITY OF SOUTHERN CALIFORNIA \\
CALIFORNIA INSTITUTE OF TECHNOLOGY & STANFORD UNIVERSITY \\
UNIVERSITY OF CALIFORNIA & UNIVERSITY OF HAWAII \\
MONTANA STATE UNIVERSITY & UNIVERSITY OF TOKYO \\
UNIVERSITY OF NEVADA, RENO & UNIVERSITY OF UTAH \\
NEW MEXICO STATE UNIVERSITY & WASHINGTON STATE UNIVERSITY \\
OREGON STATE UNIVERSITY & UNIVERSITY OF WASHINGTON
\end{tabular}

The Supporting Institutions listed above contribute to the cost of publication of this Journal, but they are not owners or publishers and have no responsibility for its content or policies.

Mathematical papers intended for publication in the Pacific Journal of Mathematics should be in typed form or offset-reproduced (not dittoed), double spaced with large margins. Please do not use built up fractions in the text of the manuscript. However, you may use them in the displayed equations. Underline Greek letters in red, German in green, and script in blue. The first paragraph must be capable of being used separately as a synopsis of the entire paper. In particular it should contain no bibliographic references. Please propose a heading for the odd numbered pages of less than 35 characters. Manuscripts, in triplicate, may be sent to any one of the editors. Please classify according to the 1991 Mathematics Subject Classification scheme which can be found in the December index volumes of Mathematical Reviews. Supply name and address of author to whom proofs should be sent. All other communications should be addressed to the managing editor, or Julie Speckart, University of California, Los Angeles, California 90024-1555.

There are page-charges associated with articles appearing in the Pacific Journal of Mathematics. These charges are expected to be paid by the author's University, Government Agency or Company. If the author or authors do not have access to such Institutional support these charges are waived. Single authors will receive 50 free reprints; joint authors will receive a total of 100 free reprints. Additional copies may be obtained at cost in multiples of 50 .

The Pacific Journal of Mathematics (ISSN 0030-8730) is published monthly except for July and August. Regular subscription rate: $\$ 190.00$ a year (10 issues). Special rate: $\$ 95.00$ a year to individual members of supporting institutions.

Subscriptions, orders for numbers issued in the last three calendar years, and changes of address should be sent to Pacific Journal of Mathematics, P.O. Box 969, Carmel Valley, CA 93924, U.S.A. Old back numbers obtainable from Kraus Periodicals Co., Route 100, Millwood, NY 10546.

The Pacific Journal of Mathematics at P.O. Box 969, Carmel Valley, CA 93924 (ISSN 0030-8730) is published monthly except for July and August. Second-class postage paid at Carmel Valley, California 93924, and additional mailing offices. Postmaster: send address changes to Pacific Journal of Mathematics, P.O. Box 969, Carmel Valley, CA 93924.

PUBLISHED BY PACIFIC JOURNAL OF MATHEMATICS, A NON-PROFIT CORPORATION Copyright $(\mathcal{1} 1993$ by Pacific Journal of Mathematics 


\section{PACIFIC JOURNAL OF MATHEMATICS}

Volume $157 \quad$ No. $2 \quad$ February 1993

Strong integral summability and the Stone-Čech compactification of the half-line

JEFF CONNOR and MARY ANNE SWARDSON

The endlich Baer splitting property

225

THEODORE GERARD FATICONI

The formal group of the Jacobian of an algebraic curve

MARGARET N. FREIJE

Concordances of metrics of positive scalar curvature

PAWEL GAJER

Explicit construction of certain split extensions of number fields and constructing cyclic classfields

STANLEY JOSEPH GURAK

Asymptotically free families of random unitaries in symmetric groups

ALEXANDRU MiHAi NiCA

On purifiable subgroups and the intersection problem

TAKASHI OKUYAMA

On the incidence cycles of a curve: some geometric interpretations

LUCIANA RAMELLA

On some explicit formulas in the theory of Weil representation

R. RANGA RAO

An analytic family of uniformly bounded representations of a free product of 373 discrete groups

JANUSZ WYSOCZAŃSKI

Errata: "Dentability, trees, and Dunford-Pettis operators on $L_{1}$ "

MARIA GIRARDI and ZHIBAO HU

Errata: "Poincaré cobordism exact sequences and characterisation" 